\title{
Datos del Archivo Arzobispal de Sevilla sobre los religiosos secularizados en dicho Arzobispado a raíz de la desamortización de Mendizábal
}

\author{
Emiliano Sánchez Pérez, OSA \\ Instituto Histórico Agustiniano (Roma)
}

\begin{abstract}
Resumen: Este trabajo pretende proporcionar datos contables, exactos o aproximados, de los religiosos exclaustrados, residentes en los conventos masculinos de Sevilla. Es un valioso soporte documental para fijar claramente la Orden o Congregación religiosa a la que pertenecían, si continuaron después como sacerdotes seculares, si todos recibieron suficiente ayuda económica del gobierno, etc, También nos guió la convicción, de que, cuando hay errores de interpretación histórica, la mejor forma de corregirla, es el recurso a las fuentes primarias. Además, la transcripción es un medio eficaz contra el peligro de desaparición o grave deterioro de las fuentes primarias.
\end{abstract}

\begin{abstract}
This work aims to provide data accounting, exact or approximate, of the religious exclaustrados, resident in the convents male of Seville. It is a valuable documentary support to clearly fix the order or religious congregation to which they belonged, if they continued then as secular priests, if they all received enough financial support from the Government, etc, also guided us the conviction, that, when there are errors in historical interpretation, the best way to correct it, is the use of primary sources. In addition, the transcription is a medium effective against the danger of disappearance or serious deterioration of the sources primary.
\end{abstract}

No es la primera vez, y confiamos que tampoco sea la última, que accedemos a este tema, pero aquí bajo la perspectiva exclusiva de transcripción documental. Usando fuentes editadas y referidos únicamente a la Orden de San Agustín, ya 
tratamos el tema hace unos años en forma sectorial. ${ }^{1}$ Ahora hacemos esta transcripción, que nos parece interesante, y que sin duda añade datos, no conocidos, a aspectos generales de Historia de la Iglesia, y más concretamente de la Iglesia Arzobispal de Sevilla en las fechas referidas en el título. En esta transcripción cultural hemos respetado escrupulosamente las características del estos dos documentos originales, entre otras razones, porque no hay razones paleográficas que justifiquen ninguna modificación, dada la cercanía cronológica de esta aportación archivística. Recordamos con auténtico cariño la gentileza humana y profesional con que fuimos tratados.Y dicho esto, lo mejor es dejar hablar a los documentos por sí mismo.

\section{Abecedario de los secularizados del Arzobispado de Sevilla}

A

1. Dn. Antonio Pino, P[resbíte]ro, Observante de San Francisco.

2. Dn. Andrés Muñoz Montoro, id.

3. Dn. Antonio Barrera, id.

4. Dn. Antonio Delgado, id.

5. Dn Alonso Souza, id.

6. Dn. Alonso Marín, id.

7. Dn. Alonso Pérez Agudo, id.

8. Dn. Alejo Solís, id.

9. Dn. Antonio Montíjano, id.

10. Dn. Antonio Romero, id.

11. Dn. Antonio del Rosal, id.

12. Dn. Andrés Jiménez, id.

13. Dn. Agustín Espinosa, id.

14. Dn. Antonio Jovar, id.

15. Dn. Antonio Gamero, id.

16. Dn. Antonio Bautista, id.

17. Dn. Antonio Salguero, id.

18. Dn. Antonio de Escamilla, Francisco Descalzo

19. Dn. Andrés Ávila, id.

20. Dn. Antonio del Caño, lego, id.

21. Dn. Andrés García, id.

22. Dn. Antonio Rendón, lego, id.

23. Dn. Andrés López, id.

24. Dn. Antonio Gamero, id.

Sánchez Pérez, Emiliano: "La Orden de San Agustín en Sevilla y el patrimonio pictórico de los conventos del Pópulo y San Agustín", en XI Congreso Internacional de Historia de la Orden de San Agustín, Roma, 2001. 
25. Dn. Antonio Marín, id.

26. Dn. Antonio Saldaña, id.

27. Dn. Antonio Romero, id.

28. Dn. Agustín Castellano, id.

29. Dn. Antonio de la Encarnación, Tercero de San Francisco.

30. Dn. Andrés Muñoz, id.

31. Dn. Antonio Corchado, id.

32. Dn. Ángel María Zamora, id.

33. Dn. Andrés María de Sevilla, Menores Capuchinos.

34. Dn. Andrés Stardales, id.

35. Dn. Antonio de Cuéllar, Agustinos Calzados.

36. Dn. Antonio Vergara, id.

37. Dn. Antonio Lique, id.

38. Dn. Antonio Carmona, id. lego.

39. Dn. Andrés Martínez Mansilla, id.

40. Dn. Anselmo Moreno, id.

41. Dn. Andrés Tenorio, id.

42. Dn. Andrés Fernández, id.

43. Dn Antonio Castillo, Agustino Descalzo.

44. Dn. Antonio González Rico, id.

45. Dn. Antonio González, id.

46. Dn. Alonso Castro, id.

47. Dn. Antonio Hernández, id.

48. Dn. Antonio Bejarano, Carmelita Calzado.

49. Dn. Antonio Lorente, id.

50. Dn. Antonio Mazuelos, id.

51. Dn. Antonio Muñoz, id.

52. Dn. Antonio Perea, id.

53. Dn. Antonio Casanbón, id.

54. Dn. Antonio Hierro, id.

55. Dn. Alonso Salguero, id.

56. Dn. Agustín de Haro, Carmelita Descalzo.

57. Dn. Alonso de la Asunción Gil, id.

58. Dn. Antonio María del Carmen, id.

59. Dn. Alonso del Espíritu Santo, id.

60. Dn. Antonio de la Concepción Alcántara, id.

61. Dn. Alonso de los Dolores García, id.

62. Dn. Antonio de Santo Tomás, id.

63. Dn. Alonso de San Juan de la Cruz, id.

64. Dn. Agustín Rodríguez, Mínimos de San Francisco de Paula.

65. Dn. Antonio Jiménez, id.

66. Dn. Antonio García, id. 
67. Dn. Acisetro Castellano, id.

68. Dn. Antonio Lagares id.

69. Dn. Alonso Hidalgo, id.

70. Dn. Andrés Ayala, id.

71. Dn. Antonio Morales, id.

72. Dn. Alonso Guillena, id.

73. Dn. Andrés Márquez, Mercedario Calzado.

74. Dn. Alonso Delgado, id.

75. Dn. Andrés Pérez, id.

76. Dn. Alonso de la Milla, id.

77. Dn. Andrés María Neto, id.

78. Dn. Antonio Setefilla López, id. Mercedario descalzo.

79. Dn. Andrés del Rosario Salazar, id.

80. Dn. Andrés de San Ramón Escudero, id. Lego.

81. Dn. Antonio de la santísima Trinidad León Roldán.

82. Dn. Alonso de la Merced, id.

83. Dn. Antonio de Jesús María Aguilar.

84. Dn. Andrés de San Cristóbal Romero, id.

85. Dn. Alonso de San José González.

86. Dn. Agustín Uribarri, Dominico.

87. Dn. Antonio López Becerra, id.

88. Dn. Antonio María Cuello, id.

89. Dn. Antonio Rodríguez, id.

90. Dn. Antonio Díaz, id.

91. Dn. Antonio Páez, id.

92. Dn. Antonio Mansera, id.

93. Dn. Alonso Morente, id.

94. Dn. Antonio Jiménez de Torre, Trinitarios.

95. Dn. Antonio García Jurado, Clérigos Regulares Menores

96. Dn. Antonio Morales, id.

97. Dn. Antonio Víbora, Monasterio de San Basilio.

98. Dn. Andrés de León, id.

99. Dn. Antonio Pozo, Francisco observante.

100. Dn. Alonso Barcia, Carmelita Calzado.

101. Antonio Rodríguez Chacón, Mercedario Calzado.

102. Dn. Antonio de Burgos, id.

103. Dn. Agustín Rodríguez Lugo, Mercedario Descalzo.

104. Dn. Antonio de la Resurrección, Carmelita Descalzo.

105. Dn. Alonso López P[resbite]ro Francisco descalzo.

106. Dn. Antonio Dorado, P[resbite]ro Dominico.

107. Dn. Antonio López, id.

108. Dn. Antonio Olivares, Agustino Calzado. 
1. Dn. Bartolomé Sánchez, Observante.

2. Dn. Bartolomé Román, id.

3. Dn. Basilio Martínez Cuadrado.

4. Dn. Blas Delgado, Tercero de San Francisco.

5. Dn. Buenaventura Fernández, Carmelita calzado.

6. Dn. Blas Carvajal, id.

7. Dn. Bartolomé Romero, id.

8. Dn. Bartolomé de Santa Teresa, Carmelita descalzo

9. Dn. Blas de Santa María, id.

10. Dn. Benito de Santa María y Marqués, id.

11. Dn. Benito González, Mínimo de San Francisco de Paula.

12. Dn. Bernardo López, id.

13. Dn. Braulio Manuel Núñez, Dominico.

14. Dn. Bernardo González, id.

15. Dn. Bernardo Martínez, Trinitario.

16. Dn. Bartolomé Fernández, Regulares Menores.

17. Dn. Blas Delgado, San Basilio.

18. Dn. Benito de la Osa, Carmelita calzado.

19. Dn. Basilio Crespo, Mercedario descalzo, lego.

\section{C}

1. Dn. Cristobal Queirós, Observante, lego.

2. Dn. Celedonio Delgado, Observante, lego.

3. Dn. Cesario de Palma, Agustino calzado.

4. Dn. Cristóbal Hidalgo, id.

5. Dn. Cayetano de Zafra, Carmelita calzado.

6. Dn. Cándido Jinoco del Castillo, id.

7. Dn. Cristóbal de Pradas, id.

8. Dn. Cristóbal de la madre de Dios, Carmelita descalzo.

9. Dn. Cristóbal Muñoz, Mínimo de San Francisco de Paula.

10. Dn. Cristóbal Carmona Caballero, Mercedario calzado.

11. Dn. Cristóbal Castellanos, id.

12. Dn. Cayetano Quijada, id.

13. Dn. Carlos de San José, Mercedario descalzo.

14. Dn. Cristóbal García, id.

15. Dn. Clemente de San Cayetano, id.

16. Dn. Cristóbal Carmona, id.

17. Dn. Cristóbal Conde, id.

18. Dn. Cristóbal soltero, P[resbite]ro. 
1. Dn. Diego Batillas, Francisco de la Observante.

2. Dn. Diego Jiménez, id.

3. Dn. Diego Fernández, id.

4. Dn. Diego Romero, id.

5. Dn. Diego Morilla, id.

6. Dn. Diego Bernal, id.

7. Dn. Diego Fernández de Cevallos, id.

8. Dn. Diego Fernández, Agustino calzado.

9. Dn. Domingo de los Ríos, id.

10. Dn. Dámaso Fernández, id.

11. Dn. Diego de Castro, id.

12. Dn. Diego Feijoo, Agustino descalzo.

13. Dn. Domingo Jiménez Avilés, id.

14. Dn. Diego de San Rafael, Carmelita descalzo.

15. Dn. Diego Romero, Mínimo.

16. Dn. Diego Parejo, id.

17. Dn. Diego Larios, lego, id.

18. Dn. Diego Falconero, Mercedario descalzo.

19. Dn. Diego Jiménez, id.

20. Dn. Diego Rejife, id.

21. Dn. Diego de la Rosa, id.

22. Dn. Diego de Belén, Mercedario descalzo.

23. Dn. Diego Ariza, Dominico.

24. Dn. Diego Sánchez, id.

25. Dn. Diego de Córdoba, Jerónimo.

26. Dn. Domingo Jiménez, Carmelita calzado.

\section{E}

1. Dn. Enrique Souza, Tercero de San Francisco.

2. Dn. Estratón Lemos, Agustino calzado.

3. Dn. Eulogio López, id.

4. Dn. Esteban Domínguez, Agustino descalzo.

5. Dn. Esteban de Santo Domingo, Mercedario descalzo.

6. Dn. Eusebio Miguel Coronado, Carmelita calzado.

7. Dn. Esteban Cabello, Francisco Observante.

\section{F}

1. Dn. Francisco Moreno, Francisco Observante.

2. Dn. Francisco Mameli Franco, id.

3. Dn. Fernando Domínguez, id. 
4. Dn. Francisco Bellido, id.

5. Dn. Francisco Fernández de Mesa, id.

6. Dn. Francisco Moreno, id.

7. Dn. Francisco Caballero, id.

8. Dn. Francisco González, id.

9. Dn. Francisco Beliño, id.

10. Dn. Francisco Díaz Borrego.

11. Dn. Francisco Páez, id.

12. Dn. Francisco Caracuel, id.

13. Dn. Francisco Segura, id.

14. Dn. Felix Díaz, id.

15. Dn. Francisco Ortiz, id.

16. Dn. Francisco Caravaca, id.

17. Dn. Francisco Delgado, id.

18. Dn. Francisco Carranza, id.

19. Dn. Francisco López, id.

20. Dn. Francisco María delValle, id.

21. Dn. Francisco Benítez, id.

22. Dn. Francisco Bello, id.

23. Dn. Francisco Jiménez, id.

24. Dn. Feliciano Sánchez Matamoros, Francisco descalzo, lego.

25. Dn. Francisco García y Esquivel, id.

26. Dn. Francisco Tejera, id.

27. Dn Francisco Cano, id.

28. Dn. Francisco Haro, id.

29. Dn. Francisco Moreno.

30. Dn. Francisco Borja palacios, Terceros de San Francisco.

31. Dn. Francisco Rivero, id.

32. Dn. Francisco de León Lara, id.

33. Dn. Francisco Javier Lucena, id.

34. Dn. Francisco Solano Sánchez, id.

35. Dn. Francisco de Atocha, id.

36. Dn. Francisco Galán, id.

37. Dn. Francisco Arévalo, id.

38. Dn. Francisco de la Concepción Esquerra, id.

39. Dn. Félix Fernández, id.

40. Dn. Francisco de Paula Funes, Agustino calzado.

41. Dn. Félix Castellano, id.

42. Dn. Francisco García Bailador, id.

43. Dn. Francisco Ruiz, id.

44. Dn. Francisco Canal, Agustino descalzo.

45. Dn. Francisco Pérez, id. 
46. Dn. Francisco Sánchez, lego, id.

47. Dn. Francisco de Paula Contreras, Carmelita calzado.

48. Dn. Feliciano Nogales, id.

49. Dn. Felipe del Toro, id.

50. Dn. Francisco de Pablo Delgado, id.

51. Dn. Francisco Javier Jiménez, id.

52. Dn. Francisco de Gracia, id.

53. Dn. Francisco de Paula Montoya, id.

54. Dn. Francisco Díaz, id.

55. Dn. Francisco de Fuentes, id.

56. Dn. Francisco Antonio de Rosas, id.

57. Dn. Francisco de Paula Gil, id.

58. Dn. Fernando de Toro, id.

59. Dn. Fernando Guerrero, id.

60. Dn. Francisco Hidalgo, id.

61. Dn. Francisco de Santo Angelo, Carmelita descalzo.

62. Dn. Francisco Pedraja, id.

63. Dn. Francisco María del Espíritu Santo, id.

64. Dn. Francisco de Paula de Santa Teresa, lego, id.

65. Dn. Francisco Polanco, id.

66. Dn. Felipe de San José, id.

67. Dn. Francisco Tirado, id.

68. Dn. Fulgencio Páez, Mínimo.

69. Dn. Francisco de Paula Sousa, id.

70. Dn. Francisco de Paula Rodríguez, corista.

71. Dn Francisco de Paula Guerra, id.

72. Dn. Fernando Sánchez, id.

73. Dn. Francisco de Guillena, id.

74. Dn. Francisco Parejo, id.

75. Dn. Francisco de Paula Guillena, id.

76. Dn. Francisco de Paula Tejeira.

77. Dn. Francisco García, lego Mercedario calzado.

78. Dn. Francisco José Liñan, id.

79. Dn. Francisco Martín Puro, id.

80. Dn. Francisco Pacheco, id.

81. Dn. Francisco Valdivieso, id.

82. Dn. Fernando Ramírez, Mercedario descalzo.

83. Dn. Fernando Mestegui, id.

84. Dn. Francisco Mila, id.

85. Dn. Francisco Fontanilla, id.

86. Dn. Francisco de la Merced, id

87. Dn. Francisco de la Cruz, id. 
88. Dn. Francisco de San José, id.

89. Dn. Francisco Muñoz, id.

90. Dn. Francisco de Paula Meneses, id.

91. Dn. Felix Delgado, Dminico.

92. Dn. Francisco de Paula Lombera, id.

93. Dn. Fernando de Silva, lego, id.

94. Dn. Francisco Ariza, id.

95. Dn. Francisco de Paula es final, id.

96. Dn. Francisco Barranco, id.

97. Dn. Francisco Reina, id.

98. Dn. Francisco de Paula Pascual, id.

99. Dn. Francisco Ramírez, id.

100. Dn. Francisco de los Santos, Clérigo menor.

101. Dn. Francisco Muñoz, Jerónimo.

102. Dn. Francisco de Rivas, Basilio.

103. Dn. Francisco de San Buenaventura, Francisco observante.

104. Dn. Francisco Pérez Valderrama, Clérigo Menor.

105. Dn. Félix Montilla, Francisco Observante.

106. Dn. Francisco de San Matías, Mercedario descalzo.

107. Dn. Francisco Montes, Mínimo.

108. Dn. Fernando González, Jerónimo.

109. Dn. Florencio Jiménez, id.

110. Dn. Francisco Ramos, P[resbíte]ro, Observante de San José.

111. Dn. Angorrila, Carmelita calzado.

112. Dn. Francisco Amador, Dominico.

\section{G}

1. Dn. Jerónimo Violencia, Francisco Observante.

2. Dn. Gabriel de Hardales, Capuchino.

3. Dn. Gregorio Lozano, Capuchino.

4. Dn. Gonzalo Pérez, Agustino calzado.

5. Dn. Gaspar Gallego, id.

6. Dn. Jerónimo Armario, id.

7. Dn. Gabriel Ortiz, id.

8. Dn. Gabriel de Cope Morales, id.

9. Dn. Gregorio Arteaga, id.

10. Dn. Gabriel de San Juan Bautista, Carmelita descalzo.

11. Dn. Jerónimo Millán, Mínimo.

12. Dn. Jerónimo Olaegui, Mercedario calzado.

13. Dn. Jerónimo Moreno, Mercedario descalzo.

14. Dn. Gregorio Jiménez, Trinitario.

15. Dn. Civiles González, Tercero. 
16. Dn. Gregorio del Carmelo, P[resbite]ro, Carmelita descalzo.

17. Dn. Gabriel Fernández, id. Dominico. ${ }^{2}$

\section{H}

1. Dn. Hermenegildo González, Tercero de San Francisco.

2. Dn. Hermenegildo Muñoz, Trinitario.

\section{I}

1. Dn. Isidoro García, Observante.

2. Dn. Ildefonso de Jesús María Prieto, Mercedario descalzo.

3. Dn. Ignacio de Sierra, Dominico.

4. Dn. Ildefonso Molina, Carmelita descalzo.

\section{J}

1. Dn. Juan Hidalgo, Observante.

2. Dn. Julián Bernal, id.

3. Dn. José María Venegas, id.

4. Dn. Juan Antonio Rodríguez, id.

5. Dn. José Marfil, id.

6. Dn. José Guerrero de ahumada, corista, id.

7. Dn. Joaquín de Vargas Machuca, id.

8. Dn. José Muraves, id.

9. Dn. Joaquín Ayala, id, lego.

10. Dn. Juan Machuca, Pro. ${ }^{3}$

11. Dn. José Carrasco, id.

12. Dn. José Ramos, id.

13. Dn. José María Fernández, id.

14. Dn. José González, id.

15. Dn. José Benítez, id.

16. Dn. José Bergillos, id.

17. Dn. Juan Sánchez Villarreal, id.

18. Dn. Juan González, id.

19. Dn. José Gutiérrez, id.

20. Dn. Juan Montero, id.

21. Dn. José Ramos, id.

22. Dn. José Patricio Sánchez, id.

23. Dn. José Quesada, id.

24. Dn. Juan González Ramos, id. 
25. Dn. Juan Mendoza, id.

26. Dn. José Álvarez, id.

27. Dn. José Domínguez, id.

28. Dn. Juan Nieto, lego.

29. Dn. José Fernández, id. Pro.

30. Dn. José Félix Roldán, id.

31. Dn. Juan Salgado, id.

32. Dn. Juan de Robles, id.

33. Dn. Juan María Romero, id.

34. Dn. Julián Romero, id.

35. Dn. Joaquín de Vega, id.

36. Dn. José Gordillo, id.

37. Dn. José del Pino, id.

38. Dn. Juan Casiano Delgado, id.

39. Dn. José Díaz Retamal, id.

40. Dn. José Romero, id.

41. Dn. Juan Rodríguez, id.

42. Dn. José Carrera, id.

43. Dn. José Becerra, id.

44. Dn. Juan González, id.

45. Dn. José Gausito, id.

46. Dn. Juan Muñoz, id.

47. Dn. José Pérez, id.

48. Dn. José Morales, id.

49. Dn. José María del Río, id.

50. Dn. José María Gandul, id.

51. Dn. Joaquín Calveto, id.

52. Dn. Juan Casal, id.

53. Dn. Juan pino de los Dolores, Francisco descalzo.

54. Dn. José Redondo, id.

55. Dn. Juan de Luque, id.

56. Dn. Juan José Galiano,id.

57. Dn. José Baena, id.

58. Dn. José Aciego del Rosario, id.

59. Dn. José MovieI, id.

60. Dn. José Cumplido, id.

61. Dn. Juan Lizcano de las Ánimas, id.

62. Dn. José de Estrada, id.

63. Dn. José de Rul, corista.

64. Dn. Juan José delValle, id.

65. Dn. Juan Galán, id.

66. Dn. Juan Gómez, id. 
67. Dn. Juan Raigadas del Rosario, ed.

68. Dn. Juan Bautista lo que el de la santísima Trinidad, id.

69. Dn. José Parrales, id.

70. Dn. José María de la Peña, id.

71. Dn. Juan de la Banda, Tercero de San Francisco.

72. Dn. José Caro, id.

73. Dn. Joaquín Rincón, id.

74. Dn. José María Vidal, lego.

75. Dn. Juan Lobo, id. Pro.

76. Dn. José García, id.

77. Dn. José Alcoba, id.

78. Dn. José Castellón, id.

79. Dn. José Escalona, id.

80. Dn. José Gómez, id.

81. Dn. José Moreno, id.

82. Dn. José Caldes, id.

83. Dn. José Moretti, id.

84. Dn. José Domínguez, id.

85. Dn. José Atienza, id.

86. Dn. Juan de Luque, id.

87. Dn. Joaquín Rodrigo, id.

88. Dn. Joaquín Pederigue, id.

89. Dn. Joaquín Arrabal, id.

90. Dn. Juan de Mela, id.

91. Dn. José María de Paradas, Menores Capuchinos.

92. Dn. José de Utrera, id.

93. Dn. Joaquín de Cádiz, id.

94. Dn. Juan Bautista de Cádiz, id.

95. Dn. Juan de Campos, Agustino calzado.

96. Dn. José Almagro, id.

97. Dn. Juan Fernández, id.

98. Dn. José Rodríguez, id.

99. Dn. Joaquín de Vargas Machuca.

100. Dn. Juan Bayacoba, id.

101. Dn. Joaquín Sánchez, id.

102. Dn. José de Luque, id.

103. Dn. Juan Monroy, id.

104. Dn. Julián José Doblado, id.

105. Dn. José Gómez, id

106. Dn. Juan de Santaella, id.

107. Dn. José García Parra, id.

108. Dn. Joaquín Rebollo, id. 
109. Dn. José Medina, lego Agustino descalzo.

110. Dn. Juan Padilla, Pro.

111. Dn. José Luque, id.

112. Dn. José Ávila, id.

113. Dn. Juan Camarero, id.

114. Dn. Juan Castañeda, Carmelita calzado.

115. Dn. Juan de Varga, id.

116. Dn. Joaquín Iglesias, id.

117. Dn. José Gadeo Escudero, subdiácono, id.

118. Dn. Juan de la Rosa, id.

119. Dn. José Pavón, id.

120. Dn. José Serrano, id.

121. Dn. Joaquín Feijoo, id.

122. Dn. Juan Nicolás Pérez, id.

123. Dn. José Sánchez Nuñez.

124. Dn José Durán, id.

125. Dn. José Cardenal, id.

126. Dn. José de Ayora, id.

127. Dn. Juan Martín Galán, id.

128. Dn. José Martín García, id.

129. Dn. Juan Romero, id.

130. Dn. Juan Puro Bejarano, id.

131. Dn. José Caballero, id.

132. Dn. José Sáenz, id.

133. Dn. José Blanco, id.

134. Dn. Juan Iglesias, id.

135. Dn. José Mateos, id.

136. Dn. Juan Gómez, id.

137. Dn. Juan José Vinuesa, id.

138. Dn. Juan María de Gracia, subdiácono, Carmelita descalzo.

139. Dn. Juan de Santa María Bejarano, id.

140. Dn. José María de la Santísima Trinidad Ballesteros.

141. Dn. Juan del Espíritu Santo, id.

142. Dn. Juan de Santa Teresa, id.

143. Dn. Juan de la Soledad, id.

144. Dn. José de San Cayetano, id.

145. Dn. Juan de San José.

146. Dn. José Rodríguez, Mínimo de San Francisco de Paula.

147. Dn. Juan Bautista Castellanos, id.

148. Dn. José Miguel Bermúdez, id.

149. Dn. José González, lego, id.

150. Dn. José Jiménez, Pro, id. 
151. Dn. José Cevallos, lego, id.

152. Dn. José Amores, Pro, id.

153. Dn. Jacoba (sic) Iglesias, id.

154. Dn. Juan Rodríguez, id.

155. Dn. José Cabrera, id.

156. Dn. Juan Pérez, Mercedario calzado.

157. Dn. Juan Díaz, Guzmán, id.

158. Dn. Joaquín Navarro, id.

159. Dn. Juan Siriaco, id.

160. Dn. José Oliva, id.

161. Dn. José Larios, id.

162. Dn. José García López, id.

163. Dn. Joaquín Magnone, id.

164. Dn. José de León, id.

165. Dn. Juan Sánchez, id.

166. Dn. Juan Jaime, id.

167. Dn. Juan Menduiña, id.

168. Dn. José Paus, id.

169. Dn. José María de Jesús Recacha, Mercedario descalzo. ${ }^{4}$

170. Dn. José Fuentes, id.

171. Dn. José Ramos, id.

172. Dn. Juan de San Arcadio, id.

173. Dn. Juan de las Mercedes, id.

174. Dn. José del santísimo Sacramento, id.

175. Dn. Juan Junieres, id.

176. Dn. José de San Cayetano, id.

177. Dn. Juan de Santa Rita, id.

178. Dn. Juan Cavas, id.

179. Dn. José de la Aurora Chacón, id.

180. Dn. Juan Caro, id.

181. Dn. Juan de las Angustias, id.

182. Dn. Juan de la Merced León Roldán, id.

183. Dn. José de la merced, id.

184. Dn. Juan de San José Caballero, id.

185. Dn. Juan de San Ramón Roldán, id.

186. Dn. Juan de San Buenaventura Alacha, id.

187. Dn. José de las Nieves Rojas, id.

188. Dn. José de San Agustín Pitares, id.

189. Dn. Juan de Santa Mariana Angovilla, id.

4 En realidad se lee claramente "Mercenario", que es impensable que existieran estos personajes en los conventos. 
190. Dn. Julián de la Cinta, id.

191. Dn. Juan Aniceto, id.

192. Dn. José se San Serapio, id.

193. Dn. Cuando los Dolores Pérez, id.

194. Dn. José de los Reyes y Vargas, id.

195. Dn. José del Santísimo Sacramento, id.

196. Dn. José de San Leonardo, id.

197. Dn. Juan de San Cayetano Romo, id.

198. Dn. José Castellano de San Cayetano

199. Dn. José Villatoro, id.

200. Dn. Juan de los Dolores Gálvez, id.

201. Dn. José García de la Santísima Trinidad, id.

202. Dn. José Rodríguez de la Soledad, id.

203. Dn. Jacinto del Rosario, id.

204. Dn. Juan de los Remedios, id.

205. Dn. José María de San Cayetano Martínez, id.

206. Dn. José de San Rafael, id.

207. Dn. José del Pino, id.

208. Dn. José Mateos, Dominico.

209. Dn. José Manuel, Subdiacono.

210. Dn. José Gil, Pro.

211. Dn. José Garrido, lego, id.

212. Dn. José Joaquín Ojeda, id.

213. Dn. José Ortigosa, id.

214. Dn. Joaquín Martínez, id.

215. Dn. Juan Ramos, id.

216. Dn. Juan Duarte, id.

217. Dn. Juan Cano, id.

218. Dn. Juan José Perez, lego, id.

219. Dn. José Mesa Jineto, id.

220. Dn. Juan García, id.

221. Dn. José Alcalde, Pro. id.

222. Dn. Juan de Lora, id.

223. Dn. Juan Moreno, id.

224. Dn. José de Robles, id.

225. Dn. José Gómez, id.

226. Dn. José María Martínes, id.

227. Dn. José Gálvez, id.

228. Dn. José Sanchez López Copero, id.

229. Dn. Juan Jiménez, Trinitarios.

230. Dn. Juan Domínguez, id.

231. Dn. José Creci, id. 
232. Dn. José Suárez, id.

233. Dn. José Guzmán, Clérigo regular menor.

234. Dn. José Ponce, id.

235. Dn. José Molina, Jerónimo.

236. Dn. Juan de Calatrava, id.

237. Dn. José de Peña, id.

238. Dn. José Montes, id.

239. Dn. José Jiménez, Basilio.

240. Dn. Juan Fuentes, id.

241. Dn. José María Aragón, id.

242. Dn. Juan Galán y Ruiz, Francisco observante.

243. Dn. Juan Bautista Contreras, id.

244. Dn. José Sánchez, id.

245. Dn. Juan Gómez, Dominico.

246. Dn. José Barro, lego Francisco observante.

247. Dn. Juan Ortiz, id. id.

248. Dn. Joaquín María Tejeira, Pro. id.

249. Dn. José de San Pedro, Mercedario descalzo.

250. Dn. José de la Misericordia, id.

251. Dn. Juan Bravo, Agustino Calzado.

252. Dn. Juan González, Pro. Observante de San Francisco.

253. Dn. Joaquín Recio, Pro. id.

254. Dn. Juan Grueso Peña, id. Dominico.

255. Dn. Juan Amador, id. id.

256. Dn. José López, id. id.

257. Dn. José de Jesús María, Carmelita descalzo.

258. Dn. Juan de los Dolores, id. id.

\section{L}

1. Dn. Luis de León, Observante.

2. Dn. Lorenzo Díaz, lego id.

3. Dn. Luis Pinto, Pro. id.

4. Dn. Lorenzo Serrano, Francisco observante.

5. Dn. Luis Cruz, id.

6. Dn. Lucas Moreno, Tercero de San Franciscocero.

7. Dn. Librado de San José Racero, id.

8. Dn. Luis Rodríguez, Agustino calzado.

9. Dn. Leonardo Salazar, Agustino descalzo.

10. Dn. Lorenzo Coso Carmelita calzado.

11. Dn. Luis González, id.

12. Dn. Lorenzo de Lora, id.

13. Dn. Luis Galindo, id. 
14. Dn. Lorenzo Romero, id.

15. Dn. Lorenzo Moyas.

16. Dn Luis Loza, id.

17. Dn. Luis Calzo, Carmelita descalzo

18. Dn. Leonardo de San Agustín, id.

19. Dn. Lorenzo de la santísima Trinidad Hidalgo, mercedario, ${ }^{5}$ Pro. id.

20. Dn. Luis Loigorría, id.

21. Dn. Lorenzo Carmona, Dominico.

22. Dn. Lucas Gutiérrez, id.

23. Dn. Lorenzo Villamor, id.

24. Dn. Lorenzo Gonzalez Antonino.

\section{$\mathbf{M}$}

1. Dn. Manuel Rodríguez, Observante.

2. Dn. Manuel Zoilo, id.

3. Dn. Manuel Rosales, id.

4. Dn. Manuel Díaz, id.

5. Dn. Miguel García, id.

6. Dn. Manuel Delgado, id, lego.

7. Dn. Manuel Calle, Pro. id.

8. Dn. Manuel Hernández, id. Pro.

9. Dn. Manuel Fernández, id.

10. Dn. Manuel Vizcaíno, id.

11. Dn. Miguel Antonio Ruiz y Pedrosa, id.

12. Dn. Manuel Ramos, id.

13. Dn. Manuel Marrufo, id.

14. Dn. Miguel Sánchez, id.

15. Dn. Manuel Monge, id.

16. Dn. Manuel Castro, id.

17. Dn. Manuel Labrador, Francisco descalzo.

18. Dn. Manuel de Fiesda, id.

19. Dn. Miguel de Campo, id.

20. Dn. Miguel García de la Aurora.

21. Dn. Manuel Lastaizo, Tercero de San Francisco.

22. Dn. Manuel Cuadrado, id.

23. Dn. Manuel Cornello, id.

24. Dn. Manuel Aldana, id.

25. Dn. Manuel Morales, id.

26. Dn. Manuel María de Hardaley, Menores capuchinos.

27. Dn. Mariano de la isla Rojas, id.

5 De nuevo escribe"mercenario". 
28. Dn. Miguel Aguilar, Agustino calzado.

29. Dn. Manuel Brunengue, id.

30. Dn. Manuel Menbiela, id.

31. Dn. Manuel campo, Agustino descalzo.

32. Dn. Manuel Gutiérrez, id.

33. Dn. Manuel de Stucerva, id.

34. Dn. Mariano Guzmán, id.

35. Dn Manuel moreno, id.

36. Dn. Miguel Toscano, id.

37. Dn. Manuel Alcaide, id.

38. Dn. Manuel Gutiérrez, id. Carmelita calzado.

39. Dn. Mariano Hernández, lego, id.

40. Dn. Martín García, id.

41. Dn. Martín de San José López, Carmelita descalzo.

42. Dn. Mateo de Jesús María León, id.

43. Dn. Manuel de San Ildefonso Rodríguez, id.

44. Dn. Manuel de Santa María Magdalena, id.

45. Dn. Miguel Navarro, id.

46. Dn. Mateo de Nuestra Señora del Carmen, lego.

47. Dn. Manuel de San José, id. id.

48. Dn. Miguel del Espíritu Santo, id. Pro.

49. Dn. Manuel Sarmiento, Mínimo de San Francisco de Paula.

50. Dn. Miguel Ruiz, id.

51. Dn. Manuel Escudero, Mercedario calzado.

52. Dn. Mariano García de Tejada, Mercedario descalzo.

53. Dn. Miguel de ser Antonio López, id.

54. Dn. Miguel Roldán, id.

55. Dn. Mauricio Corona y Setefilla, id.

56. Dn. Manuel del Espíritu Santo.

57. Dn. Manuel Astorga, id.

58. Dn. Miguel Curiel, id.

59. Dn. Manuel María de la Concepción, id.ç

60. Dn. Manuel de San Bernardo, id.

61. Dn. Manuel de Jesús María Ortiz, id.

62. Dn. Miguel Ignacio del Rosario, id.

63. Dn. Manuel Ponce, id.

64. Dn. Manuel Carmona, Dominico.

65. Dn. Manuel Buendía, id.

66. Dn. Manuel Navarro, id.

67. Dn. Manuel Nieto, id.

68. Dn. Manuel Suero, lego Tinitario.

69. Dn. MiguelVázquez, Clérigo Menor. 
70. Dn. Manuel Verdejo, Basilio.

71. Dn. Manuel Josime de San Juan de Dios.

72. Dn. Manuel Ruiz, Francisco observante.

73. Dn. Miguel de la Concepción, Mercedario descalzo

74. Dn. Miguel Vallejo, Carmelita calzado.

75. Dn. Miguel González, Dominico.

76. Dn. Manuel de Luque, Trinitario.

77. Dn. Manuel Pérez, Pro. Observante.

78. Dn. Martín Cabello, Pro. Mínimo.

79. Dn. Mateo de San Miguelm Pro. Merced descalzo.

80. Dn. Manuel de Luna, Carmelita descalzo.

81. Dn. Mariano González, Antonino.

82. Sor. María de San Joaquín, Redin en Cañete la Rl (sic).

\section{$\mathbf{N}$}

1. Dn. Nicolás Javier, Observante.

2. Dn. Nicolás Barrera, id.

3. Dn. Nicolás Carretero, Agustino calzado.

4. Dn. Nicolás Torres, Carmelita descalzo.

5. Dn. Nicómedes Fernández, mercedario descalzo.

\section{$\mathbf{P}$}

1. Dn. Pedro Gil, Observante.

2. Dn. Pedro Hidalgo, id.

3. Dn. Pablo Sánchez, id.

4. Dn. Peregrino Malcampo, Tercero de San Francisco.

5. Dn. Pascual Domínguez, Carmelita descalzo.

6. Dn. Pedro José Rovira, id.

7. Dn. Pedro Libora, id.

8. Dn. Policarpo Bancalero, id.

9. Dn. Pero Rico, id.

10. Dn. Pablo María de San José, id. [Cermelita] Descalzo.

11. Dn. Pedro Mardelo, id.

12. Dn. Pedro de la Santísima Trinidad, id.

13. Dn. Pablo María de San José, id.

14. Dn. Pedro del Carmelo, lego id.

15. Dn. Pablo Molina, Pro. id.

16. Dn. Pascual Salvador, Mercedario calzado.

17. Dn. Pedro Cabrera, id.

18. Dn. Pedro Menguiano, id.

19. Dn. Pedro Setefilla, id Descalzo. 
20. Dn. Pedro de los Dolores, id.

21. Dn. Pedro de San Cayetano, id.

22. Dn. Pablo de Jesús María Gómez, id.

23. Dn. Pedro del Rosario, id.

24. Dn. Pedro de Santa Rosa, id.

25. Dn. Pablo Hernández, Dominico.

26. Dn. Pedro Palomar, id.

27. Dn. Pedro Suárez, id.

28. Dn. Pedro José de Cana, Trinitario.

29. Dn. Pedro de Mora, Francisco de la observante.

30. Dn. Pero Peligro, id.

31. Dn. Pedro Moreno, Pro. Observante.

\section{$\mathbf{R}$}

1. Dn. Ramón Darío, id.

2. Dn. Ramón José Domínguez, Franciscano descalzo.

3. Dn. Rafael Fernández, id.

4. Dn. Ramón Fernández, Tercero de San Francisco.

5. Dn. Rafael Galán, Agustino calzado.

6. Dn. Rafael del Río y Gironda, id.

7. Dn. Ramón Mendoza, id.

8. Dn. Ramón Ramírez, id. Descalzo.

9. Dn. Rafael Sánchez, id.

10. Dn. Ramón Caro, id.

11. Dn. Rafael de Santa Teresa, Carmelita descalzo.

12. Dn. Rafael de los Dolores, id.

13. Dn. Roque González, Mínimo de San Francisco de Paula.

14. Dn. Rafael Bustillos, Mercedario calzado.

15. Dn. Ramón de la Puente, id.

16. Dn. Ramón María Fuentes, id.

17. Dn. Rodrigo de San José Pacheco, id. Descalzo.

18. Dn. Romualdo Serrano, Dominico.

19. Dn. Rafael Osorio, id.

20. Dn. Ramón Valero, lego, id. Calzado.

\section{S}

1. Dn. Sebastián Cívico, Observante de San Francisco.

2. Dn. Salvador González, id.

3. Dn. Santiago María García Nieto, Franciscano descalzo.

4. Dn. Sebastián Grande y Caballero. 
5. Dn. Segundo González, Tercero de San Francisco.

6. Dn. Sebastián Prieto, id.

7. Dn. Sebastián Caballero, Carmelita descalzo.

8. Dn. Sebastián Pérez, id.

9. Dn. Sebastián Rollano, id. Descalzo.

10. Dn. Salvador López, Mercedario descalzo.

11. Dn. Santiago Márquez, id. Descalzo.

12. Dn. Sebastián Fernández, Dominico.

13. Dn. Simón Jurado, id.

\section{$\mathrm{T}$}

1. Dn. Tomás Bueno, Francisco observante.

2. Dn. Tomás Gómez, id.

3. Dn. Toribio Vázquez, id. Descalzo.

4. Dn. Tomás del Castillo, Agustino descalzo.

5. Dn. Tomás de Jesús María, Carmelita descalzo.

6. Dn. Tomás García, Francisco observante.

\section{V}

1. Dn. Vicente Belmonte, Francisco observante.

2. Dn. Vicente Pascual, id.

3. Dn. Ventura García, id. Descalzo.

4. Dn. Vicente de la Encarnación, Mercedario descalzo.

ARCHIVO DEL ARZOBISPADO DE SEVILLA. Regulares, Leg. 15, Exp.1 


\section{Exclaustrados que han recibido pagos por la Junta 1836 - 1837}

\section{Dominicos. San Pablo de Sevilla}

D. José Pimental, corista 3 r[eale]s diarios.

El 27 de septiembre de 1836 se le pagó en virtud de decreto del Sr. intendente de la misma fecha, la pensión que le corresponde de este 1 de mayo de este año hasta hoy por ser nacional movilizado $: 450$.

El 11 de noviembre se le pagó desde 28 de septiembre hasta dicho día 11, en virtud de decreto del Sr. D. Nicolás Maestre, fecha este día : 132 .

En 29 de diciembre se le pagó la pensión que le corresponde desde 30 de noviembre hasta 7 de diciembre de 1836 por decreto del Sr. Jefe político, fecha 3 de diciembre del mismo año : 078 .

El 8 de abril de 1837 se le pagó su tensión antes de 8 de diciembre de 1836 hasta 7 de abril de 1837 en virtud del decreto de los Sres. Presidente y vocales de la junta de siete de este mes. : 0360 .

\section{Dominicos - Sto Domingo de Ceija}

D. Francisco Cea, corista de 3 r[eale]s diarios.

En 4 de noviembre de 1836 se le pagó su pensión desde el 1 de mayo hasta el 16 de octubre de dicho año, en virtud de decreto de los Sres. de la Junta fecha 4 de noviembre por haberse alistado en el escuadrón de voluntarios libres de esta capital : 507.

\section{Jerónimos de Sevilla}

Don Juan María de Flores, corista 3 r[eale]s diarios.

En 14 de noviembre de 1836 se le pagó su producción desde 1 de mayo hasta el mismo día 14 por decreto del señor Jefe político de la misma fecha 14, por ser voluntario Nacional Movilizado : 594 . 


\section{Dieguinos - San Oedro Alcántara de Sevilla}

Don José de la Torre, corista 3 reales diarios.

El 16 de noviembre de 1836 se le pagó desde el 1 de mayo hasta el 23 de junio a razón de tres pesos, y desde 24 de junio hasta 16 de noviembre de dicho año la cuarta parte de su asignación por estar sirviendo en el regimiento del príncipe $3^{\circ}$ tercero de línea, por decreto del Sr. Gobernador del día 14 de dicho mes, importando las cantidades de las dos épocas 271 pesos $17 \mathrm{~m}$ Y habiendo cobrado 275 mar[avedí]s, resulta tener percibidos mas 3 pesos 19 maravedís, que deberán descontarse de en otro pago.

El 7 de marzo de 1837 se le pagó por decreto de la junta del 6, la cuarta parte de su asignación desde el 17 de noviembre de 1836 hasta 28 de febrero de 1837 : 078.

\section{Mercedarios calzados. Sevilla}

D. Francisco Fernández de Landa, corista, 3 pesos diarios.

En 25 de noviembre de 1836 se le pagó desde el 1 de mayo de dicho año hasta el mismo día 25 de noviembre inclusive, por decreto del Sr. Jefe Político, por ser voluntario nacional movilizado : 627.

\section{$\underline{\text { San Juan de Dios. Constantina }}$}

Don José María Rodríguez cinco reales diarios.

En 18 de noviembre de 1836 se le pagó desde 1 de mayo hasta el 15 de dicho noviembre la cuarta parte de su asignación, como soldado artillero y según decreto del señor don Nicolás Maestre, de dicho día 15....... : 24528.

En 7 de enero de 1837 se le pagó la cuarta parte de la pensión, que le corresponde desde el 16 de noviembre de de 1836 hasta dicho día 7 de enero de 1837, por decreto del señor don Manuel López Cepero, de 6 de enero del mismo año :066 8 . 


\section{Tercero de San Francisco de Sevilla}

D. José Murillo, lego, las reales diarios.

En 25 de noviembre de 1836 se le pagó desde 1 de mayo hasta dicho día 25 de noviembre la cuarta parte de su asignación, que le corresponde como soldado que es del cuerpo de artillería, y según un decreto del señor jefe político de 22 de noviembre del mismo año : 15323.

En 9 de enero de 1837 se le pagó la cuarta parte de la porción que le corresponde desde 26 de noviembre de 1836 hasta 9 de enero de 1837, por decreto del Sr. jefe político de seis de dicho enero : 3425 .

\section{Dominicos de San Pablo de Sevilla}

D. José Cantón, tres reales diarios.

En 29 de noviembre de 1836 se le pagó desde 1 de mayo hasta dicho día 29 la cuarta parte de su asignación, por ser soltado en el cuerpo de artillería, y por decreto del jefe superior político del mismo día 29 de noviembre......: 15925.

\section{Terceros de Sevilla}

D. Gregorio Cantalejo, corista, pero reales diarios.

En 29 de noviembre de 1836 se le pagó desde 1 de mayo hasta dicho día 29 de noviembre la cuarta parte de su asignación, por ser soldado del regimiento de Caballería del Príncipe tercero de línea, y por decreto del Sr. jefe superior político de 28 del mismo : 15925.

\section{0 kilos de Sevilla}

don José Luis Camacho, corista, se reales diarios.

En 29 de noviembre de 1836, se le pagó desde el 1 de mayo hasta dicho día 29 de noviembre, la cuarta parte de su asignación, por ser soldado en el regimiento del príncipe tercero de línea, y por decreto del Sr. jefe superior político de 26 de noviembre del mismo año.... : 15925. 


\section{Carmelitas calzados Sanlúcar de Barrameda}

D. José Sánchez, lego, pero reales diarios.

En 23 de diciembre de 1836 se le pagó 138 reales por la cuarta parte de su asignación desde el 1 de mayo hasta 31 de octubre de dicho año, por estar sirviendo en el regimiento infantería de Ceuta, y por decreto no del Sr. Jefe superior político desde 22 de noviembre del mismo año..... : 138.

\section{Carmelitas calzados de Sevilla}

D. Martin Barranco, tres reales diarios.

En 2 de enero de 1837 se le pagó desde 1 de mayo hasta 3 de noviembre de 1936, en que ingresó en el escuadrón de voluntarios libres de esta capital, el haber que en dicho tiempo le correspondía, según decreto del Sr. jefe superior político de 24 de noviembre de 1836 : 561 .

\section{Carmelitas calzados de Sevilla}

D. Juan Obel, corista, tres reales diarios.

En 2 de enero de 1837 se le pagó la pensión que le corresponde desde el 1 de mayo hasta 4 de octubre de 1836, en que ingresó en el batallón de inmovilizados de Huelva, cuyo pago se le hizo según decreto del Sr. Jefe Superior Político de 24 de noviembre de 1836. : 471.

\section{Jerónimos de Bornos}

D. Francisco del Águila, corista, tres reales diarios.

En 11 de enero de 1837 se le pagó la cuarta parte de su pensión desde 1 de mayo hasta 31 de diciembre de 1836, como soldado del cuerpo de artillería, y por decreto del Sr. D. Manuel López Cepero de 7 de enero de dicho año 37. 18325. 


\section{Dieguinos. San Pedro Alcántara de Sevilla}

D. Francisco Saldaña, Pro, cinco reales diarios.

El 8 de febrero de 1837 se le pagó el mes de mayo de 1836 por decreto del Sr. jefe político del mismo día 8 de febrero, en razón de hallarse gravemente enfermo : 155.

\section{Francisco Sánchez Observantes. San Francisco de Estepa}

D. Juan Fita, corista, te reales diarios.

El 7 de marzo de 1837 se le pagó su asignación a razón de tres reales diarios desde el 1 de mayo hasta 10 de junio de 1836, y desde 11 de junio en que entró en el depósito de quintos hasta el 28 de febrero de 1837, la cuarta parte de de su pensión, que todo importa la cantidad de :3214.

\section{Carmelitas descalzos. Los remedios de Sevilla}

D. José Ruiz, lego, tres reales diarios.

En 14 de marzo de 1837, se le pagaron noventa y seis pesos a su apoderado D. Manuel de Señas por la cuarta parte de su asignación desde el 1 de mayo hasta el 31 de agosto de 1836, como soldado en el regimiento de caballería $4^{\circ}$ ligera, cuyo pago se le hizo en virtud de decreto de los Sres de la junta del 10 : 096 .

\section{$\underline{\text { Antoninos de Cazalla }}$}

D. José Quiroga, corista, tres reales diarios.

En 29 de marzo de 1837 se le pagó la cuarta parte de su asignación de tres reales diarios, desde 1 de mayo hasta 31 de diciembre de 1836, cuyo pago se le ha hecho por decreto de los Sres. de la junta del 21 por ser soldado del regimiento provincial de esta ciudad de Sevilla. : 78325. 


\section{Franciscos de Fregenal}

D. Primitivo Lucas, corista, te reales diarios.

En 28 De marzo de 1837, se le pagó la cuarta parte de su asignación de tres reales diarios desde 1 de mayo hasta 31 de diciembre de 1836, cuyo pago se ha hecho por decreto de los Sres. de la Junta de 28, por ser soldado del regimiento caballería segundo de línea : 13825.

\section{Franciscos de la Campana}

D. Manuel Centeno, lego, tres reales diarios.

En 29 de marzo de 1837 se le pagó la cuarta parte de su asignación de tres pesos diarios desde 1 de mayo hasta 31 de diciembre de 1836, cuyo pago se ha hecho por decreto de los Sres. de la Junta de 21 de marzo del mismo año 37. 13825.

\section{Franciscos: de Sevilla}

D. Manuel Iñiguez, presbítero, cinco reales diarios.

En 15 de octubre de 1836 se le pagaron sus haberes de los meses de Mayo junio del mismo año, por acuerdo de la junta, en atención a ser oficial de la Secretaría de la misma, importantes : 305 .

En 24 de diciembre de 1836 se le pagaron los haberes de los meses de julio agosto y septiembre de dicho año, de los cinco pesos diarios señalados por el gobierno como a exclaustrado, por acuerdo y disposición de la misma junta. : 460 .

En 24 de febrero de 1887 se le pagaron los meses de octubre, noviembre y diciembre de 1836, de su asignación de cinco pesos diarios en virtud de acuerdo de la Junta, en atención a ser oficial de la Secretaría. : 460 .

el 1 de abril del mismo año de 1837 se le pagaron sus haberes de la asignación de cinco pesos diarios correspondientes a los meses de enero febrero y marzo de dicho año por acuerdo de la Junta. 450. 


\section{Capuchinos: de Sevilla}

D. Francisco de Paula Alesón, presbítero, cinco pesos diarios.

En 15 de octubre de 1836 se le pagaron sus haberes de cinco pesos diarios señalados por el gobierno correspondiente a los meses de mayo y junio del mismo año, el orden de la Junta, en atención a ser oficial de la Secretaría de la misma : 460 .

En 24 de febrero de 1837, se le pagaron sus haberes de los expresados 5 pesos diarios, por los meses de octubre, noviembre y diciembre de 1836, en virtud de acuerdo de la Junta, atendiendo a que es Oficial de la Secretaría : 460

En $1^{\circ}$ de Abril de 1837 , se le pagaron sus haberes de los 5 pesos diarios de su asignación, correspondiente a los meses de enero, febrero y marzo, de dicho año, de orden de la Junta, en atención a ser oficial de la Secretaria de la misma : 450 .

\section{Dominicos: S. Pablo de Sevilla}

En 15 de octubre de 1836 se le pagaron los haberes de su asignación de 5 pesos diarios señalados por el gobierno correspondiente a los meses de mayo y junio de dicho año, por orden de la Junta, en atención a ser oficial de la Secretaría de la misma : 305 .

En 24 de diciembre de 1836 se le pagaron los meses de julio, agosto y septiembre de dicho año, por disposición de la Junta, atendiendo que es oficial de dicha Secretaría. : 460 .

En 24 de febrero de 1837 se le pagaron los haberes de su asignación de cinco pesos diarios pertenecientes a los meses de octubre, noviembre y diciembre de 1836, en virtud de orden de la Junta, en consideración a ser oficial de dicha Secretaría. : 460 .

En 1 de abril de 1837, se le pagaron sus haberes de cinco pesos diarios, pertenecientes a los meses de enero, febrero y marzo de dicho año, por orden de la Junta, en atención a ser oficial de la Secretaría de la misma. : 450 . 


\section{Agustinos Calzados: de Sevilla}

D. Baltasar Rodríguez, presbítero, 5 pesos diarios.

En 15 de octubre de 1836, se le pagaron los haberes de su asignación de 5 pesos diarios, correspondientes a los meses de mayo y junio de dicho año, de orden de la Junta, en atención a ser oficial de la Secretaría de la misma... : 305.

En 24 de diciembre de 1836, se le pagaron sus asignaciones de 5 pesos diarios, correspondientes a los meses de julio, agosto y septiembre de dicho año, por acuerdo de la Junta, atendiendo que es oficial de dicha Secretaría ...: 460.

En 24 de febrero de 1837, se le pagaron los haberes de su asignación de 5 pesos diarios, correspondientes a los meses de octubre, noviembre y diciembre de 1836, en virtud de que de la Junta, atendiendo a ser oficial de dicha Secretaría. : 460 .

En 1 de abril de 1837, se le pagaron sus haberes de la asignación de 5 pesos diarios, correspondientes a los meses de enero, febrero y marzo de dicho año, por orden de la Junta, en atención a ser oficial de dicha Secretaría : 450.

\section{Capuchinos: de Sevilla}

D. Joaquín Sanz, lego, tres reales diarios.

En 15 de octubre de 1836, se le pagaron sus haberes de los 3 pesos asignados por el gobierno, correspondientes a los meses de mayo y junio del mismo año por orden de la Junta, en atención a ser oficial de la Secretaría, importantes : 183.

En 24 de diciembre de 1836, se le pagaron los haberes de sus 3 pesos asignados, correspondientes a los meses de julio, agosto y septiembre del mismo año, de orden de la Junta, atendiendo a que es oficial de la Secretaría ...: 276.

En 24 de febrero de 1837, se le pagaron los haberes de sus tres pesos diarios, asignados por el gobierno, pertenecientes a los meses de octubre, noviembre y diciembre de 1836, por disposición de la Junta, en atención a ser oficial de la Secretaría de la misma : 276.

En 1 de abril de 1837, se le pagaron los haberes de sus tres pesos diarios, correspondientes a los meses de enero, febrero y marzo de dicho año, por orden de la Junta, en atención a ser oficial de su Secretaría $: 270$. 


\section{Capuchinos: de Sevilla}

D. Romualdo de Reyes, lego, tres pesos diarios.

En 15 de octubre de 1836, se le pagaron los haberes de sus 3 pesos asignados por el gobierno, correspondientes a los meses de mayo y junio de dicho año, por lo que el de la Junta, en atención a ser Portero de la Secretaría de la misma : 183.

En 23 de diciembre de 1836, se le pagaron sus haberes de los 3 pesos señalados por el gobierno, correspondientes a los meses de julio, agosto y septiembre, por disposición de la Junta, en atención a ser Portero de la Secretaría : 276.

En 24 de febrero de 1837, se le satisficieron los haberes de sus 3 pesos diarios, pertenecientes a los meses de octubre, noviembre y diciembre de 1836, por orden de la Junta, atendiendo a que es Portero de la Secretaría de la misma : 276.

En 1 de abril de 1837, se le pagaron por la Junta de los haberes de sus 3 pesos diarios, correspondientes a los meses de enero, febrero y marzo de dicho año, en consideración a ser Portero de la Secretaría de dicha junta 270 .

ARCHIVO ARZOBISPAL DE SEVILLA, Regulares, Leg. 9, Exp. 13. 